\title{
Understanding Android App Piggybacking
}

\author{
Li Li*, Daoyuan Li*, Tegawendé F. Bissyandé*, Jacques Klein*, Yves Le Traon*, David Lo ${ }^{\dagger}$, Lorenzo Cavallaro ${ }^{\ddagger}$ \\ * Interdisciplinary Centre for Security, Reliability and Trust (SnT), University of Luxembourg \\ $\dagger$ School of Information Systems, Singapore Management University \\ $\ddagger$ Information Security Group, Royal Holloway, University of London \\ firstName.lastName@uni.lu; davidlo@smu.edu.sg; Lorenzo.Cavallaro@ rhul.ac.uk
}

\begin{abstract}
The Android packaging model offers adequate opportunities for attackers to inject malicious code into popular benign apps, attempting to develop new malicious apps that can then be easily spread to a large user base. Despite the fact that the literature has already presented a number of tools to detect piggybacked apps, there is still lacking a comprehensive investigation on the piggybacking processes. To fill this gap, in this work, we collect a large set of benign/piggybacked app pairs that can be taken as benchmark apps for further investigation. We manually look into these benchmark pairs for understanding the characteristics of piggybacking apps and eventually we report 20 interesting findings. We expect these findings to initiate new research directions such as practical and scalable piggybacked app detection, explainable malware detection, and malicious code location.
\end{abstract}

\section{INTRODUCTION}

Thanks to a set of existing tools, Android apps can easily be modified by third parties [1], [2]. Malware writers can thus build on top of popular benign apps to rapidly spread new malware. Indeed, it would be more effective to simply mutate a popular benign app (e.g., by injecting some malicious code) for distributing malicious functionalities. The resulting mutant, which thus piggybacks a malicious payload, is referred to as a piggybacked app.

Fig. 1 illustrates constituting parts of a piggybacked app. The piggybacking process involves in selecting a given original app, referred to in the literature [3] as the carrier, and grafting to it a malicious code, known as the rider. The connection between carrier to rider is known as hook, which defines the point where the execution of malicious code can be triggered.

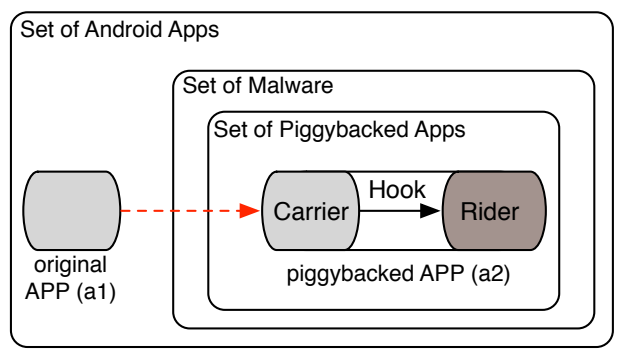

Fig. 1. Piggybacking Terminology [4].

State-of-the-art works have mainly focused on detecting piggybacked apps (or cloned apps in general) through similarity comparison [5], [6], [7], [8], [9], [10], [11], [12], [13], [14],
[15], [16], [17], [18], [19], [20], [21], [22], [23], [24], [25], [26], [27], [28], [29], [30], [31]. However, pairwise comparison based approaches are not scalable for analyzing millions of Android apps that are now available in various markets. Besides, the comparison-based approaches also require that both benign and piggybacked apps are available in the evaluated app set. Instead of a brute-force comparison, a more practical solution is to leverage semantic features collected through a thorough understanding on piggybacking scenarios to tame the problem of piggybacked apps. Indeed, understanding Android app piggybacking could help in pushing further a number of research directions: 1) Practical detection of piggybacked apps (e.g., through machine learning based predictors); 2) Explainable detection of piggybacked apps (e.g., through finegrained semantic features); and 3) Automatic localization of piggybacked malicious payloads (e.g., through graph-based analysis [32], [33]). Interested readers are encouraged to obtain more information from the journal publication (cf. [4]) of this extended abstract.

\section{APPROACH}

Our objective is to conduct a thorough dissection on piggybacked Android apps and thereby to have a deep understanding on how Android apps are piggybacked. The observed knowledge can then be used to invent advanced techniques for taming the Android app piggybacking problem. Although several approaches have been proposed to tackle this problem [34], [5], their associated datasets are not always released to public [35], [6], [31], [36]. In other words, the research on piggybacked apps is challenged by the scarcity of datasets and benchmarks. To this end, in this work, we first present an automate approach to systematically collect a set of trustable benchmarks (i.e., piggybacked apps) before conducting indepth dissection on piggybacked apps.

\section{A. Benchmark Collection}

Our collection is based on AndroZoo [37], a large repository of millions of apps crawled over several markets including the official one named Google Play. As shown in Fig. 2, the benchmark collection is mainly done in three steps. We now briefly describe them respectively.

- VirusTotal Classification. First, we collect the malicious status of Android apps through the associated anti-virus scanning reports of VirusTotal. Based on the identified 


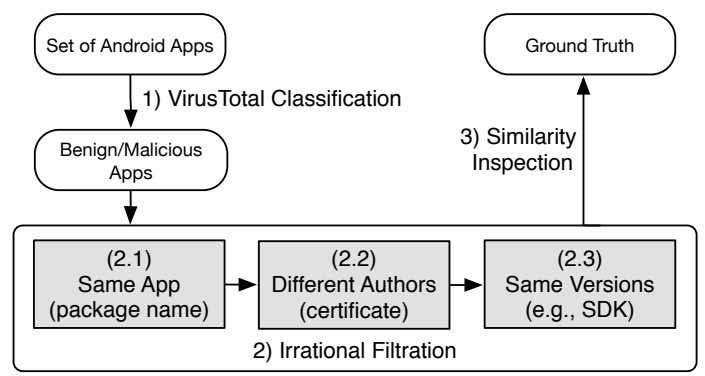

Fig. 2. The Benchmark Building Process [4].

malicious status, we divide the set of apps into two subsets: benign set and malicious set.

- Irrelevance Filtering. Second, in order to only concentrate on piggybacked app pairs, we filter out irrelevant results through a set of meta-data (including the unique app package name, app certificate and the app version) extracted from Android apps. We remind the readers that this step may miss a number of piggybacking pairs, but those that we have found will unlikely be false positives.

- Similarity Inspection. Finally, we conduct pairwise similarity comparison on the candidate pairs (remaining in the second step) to validate the correctness of piggybacked pairs. Given a pair of candidate apps $\left(a_{1}, a_{2}\right)$, we expect that the majority code of $a_{1}$ should be part of $a_{2}$ while $a_{2}$ should also include new code to constitute its malicious payload.

The aforementioned three steps allow for a conservative identification of piggybacking pairs. We would like to emphasize that our focus was not to precisely detect all piggybacking pairs. Instead, we aimed for collecting a sufficient number of accurate pairs in order to be able to dissect and understand piggybacking processes. Therefore, as indicated before, our approach may have missed a number of piggybacking pairs, but those that we have found are unlikely to be false positives.

\section{B. Piggybacking Dissection}

Based on the identified piggybacking pairs, we manually look into the difference between the original and piggybacked apps with an attempt to understand how piggybacking is done. In addition to the manual investigation, we also leverage some automated tools (e.g., Soot-based static analyzer) and scripts (e.g., Shell and Python) to facilitate our analysis. As an example, our similarity analysis approach (the similarity inspection step) is implemented in Java on top of Soot, a framework for analyzing and transforming Java/Android apps [38]. The comparison is eventually conducted at Soot's Jimple level, where Jimple is a simplified representation of Android Dalvik bytecode. The representation is conducted by Dexpler [2], which now has been integrated as a plugin into Soot.

\section{FINDINGS}

Our dissection explores several aspects of Android app piggybacking in order to answer the following three research dimensions: 1) Which app elements are manipulated by piggybackers? 2) How app functionality and behavior are impacted? and 3) Where malicious code is hooked into benign apps?

With these three research dimensions in mind, our dissection has eventually identified 20 interesting findings. Because of space limitation, we only highlight take-home messages of those findings. We recommend readers to read the detailed explanation of those findings in our journal publication [4]. The abstracted findings are as follows:

1) The realization of malicious behavior is often accompanied by a manipulation (i.e., adding/removing/replacing) of app resource files.

2) Piggybacking modifies app behavior mostly by tampering with existing original app code.

3) Piggybacked apps are potentially built in batches.

4) Piggybacking often requires new permissions to allow the realization of malicious behavior.

5) Some permissions appear to be more requested by piggybacked apps than non-piggybacked apps.

6) Piggybacking is probably largely automated.

7) Piggybacked apps overly request permissions, while leveraging permissions requested by their original apps.

8) Most piggybacked apps now include new user interfaces, implement new receivers and services, but do not add new database structures.

9) Piggybacking often consists in inserting a component that offers the same capabilities as an existing component in the original app.

10) Piggybacked apps can simply trick users by changing the launcher component in the app, in order to trigger the execution of rider code.

11) Piggybacking is often characterized by a naming mismatch between existing and inserted components.

12) Malicious piggybacked payload is generally connected to the benign carrier code via a single method call statement, making it possible to automatically locate grafted malicious payloads from piggybacked malicious apps [32], [33].

13) Piggybacking hooks are generally placed within library code rather than in core app code.

14) Injected payload is often reused across several piggybacked apps.

15) Piggybacking adds code which performs sensitive actions, often without referring to device users.

16) Piggybacking operations spread well-known malicious behavior types.

17) Piggybacked apps increasingly hide malicious actions via the use of reflection and dynamic class loading.

18) Piggybacking code densifies the overall app's call graph, while rider code can even largely exceed in size the carrier code.

19) Piggybacked app writers are seldom authors of benign apps.

20) Piggybacking code brings more execution paths where sensitive data can be leaked. 


\section{REFERENCES}

[1] R Winsniewski. Apktool: a tool for reverse engineering android apk files. URL: https://ibotpeaches.github.io/Apktool/(vi sited on 07/27/2016), 2012.

[2] Alexandre Bartel, Jacques Klein, Martin Monperrus, and Yves Le Traon. Dexpler: Converting android dalvik bytecode to jimple for static analysis with soot. In SOAP, 2012.

[3] Wu Zhou, Yajin Zhou, Michael Grace, Xuxian Jiang, and Shihong Zou. Fast, scalable detection of piggybacked mobile applications. In CODASPY, 2013.

[4] Li Li, Daoyuan Li, Tegawendé F Bissyandé, Jacques Klein, Yves Le Traon, David Lo, and Lorenzo Cavallaro. Understanding android app piggybacking: A systematic study of malicious code grafting. IEEE Transactions on Information Forensics \& Security, 2017.

[5] Li Li, Tegawendé F Bissyandé, Jacques Klein, and Yves Le Traon. An investigation into the use of common libraries in android apps. In SANER, 2016

[6] Quanlong Guan, Heqing Huang, Weiqi Luo, and Sencun Zhu. Semantics-based repackaging detection for mobile apps. In ESSoS, 2016.

[7] Li Li, Tegawendé F Bissyandé, and Jacques Klein. Simidroid: Identifying and explaining similarities in android apps. In Technical Report, 2017.

[8] Xueping Wu, Dafang Zhang, Xin Su, and WenWei Li. Detect repackaged android application based on http traffic similarity. SCN, 2015.

[9] Mingshen Sun, Mengmeng Li, and John C.S. Lui. Droideagle: seamless detection of visually similar android apps. In WiSec, 2015.

[10] Charlie Soh, Hee Beng Kuan Tan, Yauhen Leanidavich Arnatovich, and Lipo Wang. Detecting clones in android applications through analyzing user interfaces. In ICPC, 2015.

[11] Jian Chen, Manar H. Alalfi, Thomas R. Dean, and Ying Zou. Detecting android malware using clone detection. JCST, 2015.

[12] Kai Chen, Peng Wang, Yeonjoon Lee, XiaoFeng Wang, Nan Zhang, Heqing Huang, Zou Wei, and Peng Liu. Finding unknown malice in 10 seconds: Mass vetting for new threats at the google-play scale. In USENIX Security, 2015.

[13] Hugo Gonzalez, Natalia Stakhanova, and Ali A Ghorbani. Droidkin: Lightweight detection of android apps similarity. In International Conference on Security and Privacy in Communication Systems, pages 436-453. Springer, 2014.

[14] Israel J. Ruiz, Bram Adams, Meiyappan Nagappan, Steffen Dienst, Thorsten Berger, and Ahmed E. Hassan. A large scale empirical study on software reuse in mobile apps. IEEE Software, 2014.

[15] Mario Linares-Vasquez, Andrew Holtzhauer, Carlos Bernal-Cardenas, and Denys Poshyvanyk. Revisiting android reuse studies in the context of code obfuscation and library usages. In MSR, 2014.

[16] Kai Chen, Peng Liu, and Yingjun Zhang. Achieving accuracy and scalability simultaneously in detecting application clones on android markets. In ICSE, 2014.

[17] Nicolas Viennot, Edward Garcia, and Jason Nieh. A measurement study of google play. In SIGMETRICS, 2014.

[18] Yury Zhauniarovich, Olga Gadyatskaya, Bruno Crispo, Francesco La Spina, and Ermanno Moser. Fsquadra: fast detection of repackaged applications. In IFIP Annual Conference on Data and Applications Security and Privacy, pages 130-145. Springer, 2014.

[19] Fangfang Zhang, Heqing Huang, Sencun Zhu, Dinghao Wu, and Peng Liu. Viewdroid: Towards obfuscation-resilient mobile application repackaging detection. In WiSec, 2014.
[20] Xin Sun, Yibing Zhongyang, Zhi Xin, Bing Mao, and Li Xie. Detecting code reuse in android applications using component-based control flow graph. In IFIP SEC, 2014.

[21] Martina Lindorfer, Stamatis Volanis, Alessandro Sisto, Matthias Neugschwandtner, Elias Athanasopoulos, Federico Maggi, Christian Platzer, Stefano Zanero, and Sotiris Ioannidis. Andradar: Fast discovery of android applications in alternative markets. In DIMVA, 2014.

[22] Su Mon Kywe, Yingjiu Li, Robert H. Deng, and Jason Hong. Detecting camouflaged applications on mobile application markets. In ICISC, 2014.

[23] Min Zheng, Mingshen Sun, and John C.S. Lui. Droidanalytics: A signature based analytic system to collect, extract, analyze and associate android malware. In TrustCom, 2013.

[24] Clint Gibler, Ryan Stevens, Jonathan Crussell, Hao Chen, Hui Zang, and Heesook Choi. Adrob: Examining the landscape and impact of android application plagiarism. In MobiSys, 2013.

[25] Wu Zhou, Yajin Zhou, Xuxian Jiang, and Peng Ning. Detecting repackaged smartphone applications in third-party android marketplaces. In CODASPY, 2012.

[26] Steve Hanna, Ling Huang, Edward Wu, Saung Li, Charles Chen, and Dawn Song. Juxtapp: a scalable system for detecting code reuse among android applications. In DIMVA, 2012.

[27] Jonathan Crussell, Clint Gibler, and Hao Chen. Attack of the clones: detecting cloned applications on android markets. In ESORICS, 2012.

[28] Anthony Desnos. Android: Static analysis using similarity distance. In System Science (HICSS), 2012 45th Hawaii International Conference on, pages 5394-5403. IEEE, 2012.

[29] Rahul Potharaju, Andrew Newell, Cristina Nita-Rotaru, and Xiangyu Zhang. Plagiarizing smartphone applications: Attack strategies and defense techniques. In ESSoS, 2012.

[30] Israel J. Ruiz, Meiyappan Nagappan, Bram Adams, and Hassan Ahmed E. Understanding reuse in the android market. In ICPC, 2012.

[31] Haoyu Wang, Yao Guo, Ziang Ma, and Xiangqun Chen. Wukong: A scalable and accurate two-phase approach to android app clone detection. In ISSTA, 2015.

[32] Li Li, Daoyuan Li, Tegawendé F Bissyandé, Jacques Klein, Haipeng Cai, David Lo, and Yves Le Traon. Automatically locating malicious packages in piggybacked android apps. In Technical Report, 2017.

[33] Li Li, Daoyuan Li, Tegawendé F Bissyandé, David Lo, Jacques Klein, and Yves Le Traon. Ungrafting malicious code from piggybacked android apps. Technical Report, 2016.

[34] Li Li, Tegawendé F Bissyandé, Mike Papadakis, Siegfried Rasthofer, Alexandre Bartel, Damien Octeau, Jacques Klein, and Yves Le Traon. Static analysis of android apps: A systematic literature review. Technical report, SnT, 2016.

[35] Ke Tian, Danfeng (Daphne) Yao, Barbara G. Ryder, and Gang Tan. Analysis of code heterogeneity for high-precision classification of repackaged malware. In MoST@S\&P $(W), 2016$.

[36] Yuru Shao, Xiapu Luo, Chenxiong Qian, Pengfei Zhu, and Lei Zhang. Towards a scalable resource-driven approach for detecting repackaged android applications. In ACSAC, 2014.

[37] Kevin Allix, Tegawendé F Bissyandé, Jacques Klein, and Yves Le Traon. Androzoo: Collecting millions of android apps for the research community. In $M S R, 2016$.

[38] Patrick Lam, Eric Bodden, Ondrej Lhoták, and Laurie Hendren. The soot framework for java program analysis: a retrospective. In CETUS, 2011 . 